Draft version December 25, 2010

Preprint typeset using $\mathrm{LT}_{\mathrm{E}} \mathrm{X}$ style emulateapj v. 8/13/10

\title{
POWER OF OBSERVATIONAL HUBBLE PARAMETER DATA: A FIGURE OF MERIT EXPLORATION
}

\author{
Cong MA ${ }^{1}$ And Tong-JIE Zhang ${ }^{1,2}$ \\ Draft version December 25, 2010
}

\begin{abstract}
We use simulated Hubble parameter data in the redshift range $0 \leq z \leq 2$ to explore the role and power of observational $H(z)$ data in constraining cosmological parameters of the $\Lambda$ CDM model. The error model of the simulated data is empirically constructed from available measurements and scales linearly as $z$ increases. By comparing the median figures of merit calculated from simulated datasets with that of current type Ia supernova data, we find that as many as 64 further independent measurements of $H(z)$ are needed to match the parameter constraining power of SNIa. If the error of $H(z)$ could be lowered to $3 \%$, the same number of future measurements would be needed, but then the redshift coverage would only be required to reach $z=1$. We also show that accurate measurements of the Hubble constant $H_{0}$ can be used as priors to increase the $H(z)$ data's figure of merit.
\end{abstract}

Subject headings: cosmological parameters - dark energy — distance scale - methods: statistical

\section{INTRODUCTION}

The expansion of the Universe can be quantitatively studied using the results from a variety of cosmological observations, for example the mapping of the cosmic microwave background (CMB) anisotropies (Spergel et al. 2007. Komatsu et al. 2010), the measurement of baryon acoustic oscillation (BAO) peaks (Eisenstein et al. 2005 Percival et al. 2010), the linear growth of large-scale structures (LSS) (Wang \& Tegmark 2004), strong gravitational lensing (Yang \& Chen 2009), and measurements of "standard candles" such as the redshift-distance relationship of type Ia supernovae (SNIa) (Riess et al. 1998 Hicken et al. 2009b) and gamma-ray bursts (GRBs) (Ghirlanda et al. 2004 Li et al. 2008). Among the various observations is the determination of the Hubble parameter $H$ which is directly related to the expansion history of the Universe by its definition: $H=\dot{a} / a$, where $a$ denotes the cosmic scale factor and $\dot{a}$ is its rate of change with respect to the cosmic time. In practice, the Hubble parameter is usually measured as a function of the redshift $z$, and the redshift is related to $a$ by the formula $a(t) / a\left(t_{0}\right)=1 /(1+z)$ where $t_{0}$ is the current time that is taken to be a constant. In the rest of this paper we will use the abbreviation "OHD" for observational $H(z)$ data.

Though not directly observable, $H(z)$ can nevertheless be deduced from various observational data, such as cosmological ages ("standard clocks") or sizes ("standard rods"). The former method has been illustrated by Jimenez \& Loeb (2002) and leads to OHD found from differential ages of galaxies. The latter method has been discussed by Blake \& Glazebrook (2003) and Seo \& Eisenstein (2005), which leads to $H(z)$ found from BAO peaks.

Moreover, the Hubble parameter as a quantitative measure of the cosmic expansion rate is closely related to cosmological distances. In particular, it can be recon-

\footnotetext{
tjzhang@bnu.edu.cn

${ }^{1}$ Department of Astronomy, Beijing Normal University, Beijing 100875, China

${ }^{2}$ Center for High Energy Physics, Peking University, Beijing 100871, China
}

structed from the luminosity distances of SNIa (Wang \& Tegmark 2005; Shafieloo et al. 2006; |Mignone \& Bartelmann 2008) and GRBs (Liang et al. 2010). On the other hand, given the aforementioned availability of independent determination of $H(z)$, and the expectation of more available data in the future, we are interested in the comparison of OHD and SNIa data in terms of their respective merits in constraining cosmological parameters. This interest of ours is expressed by the following two questions: Can future observational determinations of the Hubble parameter be used as a viable alternative to current SNIa data? If so, how many more datapoints are needed so that the cosmological parameter constraints obtained from $H(z)$ data are as good as those obtained from SNIa distance-redshift relations?

We attempt to illustrate possible answers to these two questions via an exploratory, statistical approach. This paper is organized as follows: we first briefly summarize the current status of available OHD results in Section 2. Next, we show how the simulated $H(z)$ datasets are used in our exploration in Section 3. and present the results from the simulated data in Section 4. In Section 5 we turn to the data expected from future observation programs. Finally, in Section 6 we discuss the limitation and implications of our results.

\section{AVAILABLE HUBBLE PARAMETER DATASETS}

Currently the amount of available $H(z)$ data is scarce compared with SNIa luminosity distance data. Jimenez et al. (2003) first obtained one $H(z)$ data point at $z \approx$ 0.1 from observations of galaxy ages (henceforward the "JVTS03" dataset). Simon et al. (2005) further obtained 8 additional $H(z)$ values up to $z=1.75$ from the relative ages of passively evolving galaxies (henceforward "SVJ05") and used it to constrain the redshift dependency of dark energy potential. Stern et al. (2010a) obtained an expanded dataset (henceforward "SJVKS10") and combined it with CMB data to constrain dark energy parameters and the spatial curvature. Besides $H(z)$ determinations from galaxy ages, observations of BAO peaks have also been used to extract $H(z)$ values at low redshift (Gaztañaga et al. 2009, henceforward 
Table 1

Currently Available $H(z)$ Datasets

\begin{tabular}{lccl}
\hline \hline \multicolumn{1}{c}{$z$} & SVJ05 & SJVKS10 & GCH09 $^{\mathrm{a}}$ \\
\hline $0.09^{\mathrm{b}}$ & $69 \pm 12$ & $69 \pm 12$ & $\ldots$ \\
0.17 & $83 \pm 8.3$ & $83 \pm 8$ & $\ldots$ \\
$0.24_{-0.09}^{+0.06}$ & $\ldots$ & $\ldots$ & $79.69 \pm 2.65$ \\
0.27 & $70 \pm 14$ & $77 \pm 14$ & $\ldots$ \\
0.4 & $87 \pm 17.4$ & $95 \pm 17$ & $\ldots$ \\
$0.43_{-0.03}^{+0.04}$ & $\ldots$ & $\ldots$ & $86.45 \pm 3.68$ \\
0.48 & $\ldots$ & $97 \pm 62$ & $\ldots$ \\
0.88 & $117 \pm 23.4$ & $90 \pm 40$ & $\ldots$ \\
0.9 & $\ldots$ & $117 \pm 23$ & $\ldots$ \\
1.3 & $168 \pm 13.4$ & $168 \pm 17$ & $\ldots$ \\
1.43 & $177 \pm 14.2$ & $177 \pm 18$ & $\ldots$ \\
1.53 & $140 \pm 14$ & $140 \pm 14$ & $\ldots$ \\
1.75 & $202 \pm 40.4$ & $202 \pm 40$ & $\ldots$ \\
\hline
\end{tabular}

Note. - $H(z)$ figures quoted in this table are in the units of $\mathrm{km} \mathrm{s}^{-1} \mathrm{Mpc}^{-1}$.

a Uncertainties include both statistical and systematic errors: $\sigma^{2}=\sigma_{\text {sta }}^{2}+\sigma_{\text {sys }}^{2}$. See Section 2.4 and Table 3 of Gaztañaga et al. (2009).

b Data in this row are taken from JVTS03.

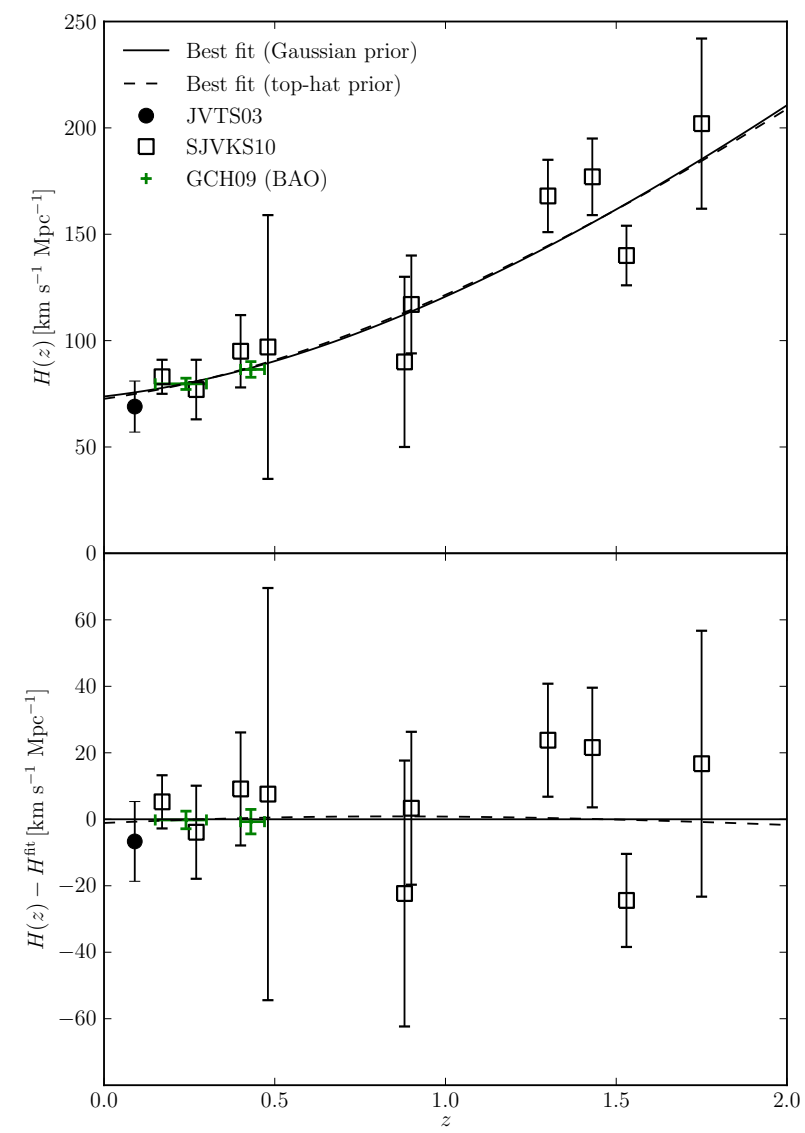

Figure 1. Full OHD set and best-fit $\Lambda$ CDM models. The top panel shows the dataset and fit results. The bottom panel shows the residuals with respect to the best-fit model with Gaussian prior on $H_{0}$. The $H_{0}$ priors are described in Section 3.2

"GCH09"). These datasets are summarized in Table 1 and displayed in Figure 1

These datasets have seen wide application in cosmological research. In addition to those mentioned above, Yi \& Zhang (2007) first used the SVJ05 dataset to con- strain cosmological model parameters. Samushia \& Ratra (2006) also used the data to constrain parameters in various dark energy models. Their results are in consistence with other observational data, in particular the SNIa. Besides parameters constraints, OHD can also be used as an auxiliary model selection criterion ( $\mathrm{Li}$ et al. 2009).

In this paper, the OHD sets used are taken from the union of JVTS03, GCH09, and SJVKS10. The SVJ05 dataset, having been replaced by SJVKS10, is no longer used, and is listed in Table 1 for reference only. Using these datasets, we find the parameter constraints for a non-flat $\Lambda \mathrm{CDM}$ universe $\Omega_{\mathrm{m}}=0.37_{-0.16}^{+0.15}$ and $\Omega_{\Lambda}=0.93_{-0.29}^{+0.25}$ assuming the Gaussian prior $H_{0}=74.2 \pm$ $3.6 \mathrm{~km} \mathrm{~s}^{-1} \mathrm{Mpc}^{-1}$ suggested by Riess et al. (2009). We also use a conservative, "top-hat" prior on $H_{0}$, namely a uniform distribution in the range $[50,100]$, and obtained $\Omega_{\mathrm{m}}=0.34_{-0.27}^{+0.20}$ and $\Omega_{\Lambda}=0.86_{-064}^{+0.44}$. These $H_{0}$ priors are discussed in detail in Section 3.2 , and their effects on the parameter constraints are illustrated in Section 4.

\section{PARAMETER CONSTRAINT WITH SIMULATED DATASETS}

In the current absence of more OHD, we turn to simulated $H(z)$ datasets in the attempt to explore the answer to the questions raised in Section 1 . To proceed with our exploration, we must prepare ourselves with a) a way of generating simulated $H(z)$ datasets, b) an "evaluation" model (or class of models) of cosmic expansion in which parameter constraint is performed using the simulated data, and c) a quantified measure of the datasets' ability of tightening the constraints in the model's parameter space, i.e. a well-defined "figure of merit" (FoM). These topics are discussed in detail in the rest of this section.

\subsection{Generation of Simulated Datasets}

Our simulated datasets are based on a spatially flat $\Lambda$ CDM model with $\Omega_{\mathrm{m}}=0.27$ and $\Omega_{\Lambda}=0.73$. This fiducial model is consistent with the 7-year Wilkinson Microwave Anisotropy Probe (WMAP) (Komatsu et al. 2010), the BAO (Percival et al. 2010), and SNla (Hicken et al. 2009b) observations. Therefore, it summarizes our current knowledge about the recent history of cosmic expansion fairly well. In this fiducial model the Hubble parameter is expressible as a function of redshift $z$ by the simple formula

$$
H_{\text {fid }}(z)=H_{0} \sqrt{\Omega_{\mathrm{m}}(1+z)^{3}+\Omega_{\Lambda}}
$$

where $H_{0}$ is the Hubble constant.

The modelling of the observational data's deviations from the fiducial model, as well as the statistical and systematic uncertainties of the data, can be rather difficult. For SNIa, there are planned projects such as the WideField Infrared Survey Telescope (WFIRST ${ }^{3}$ ) with welldefined redshift distribution of targets (Aldering et al. 2004) and uncertainty model (Kim et al. 2004 Huterer 2009 based on which simulated data can be generated. However, this is not true for OHD, as there have not been a formal specification of future observational goals known to the authors. Consequently, we have to work

\footnotetext{
3 http://wfirst.gsfc.nasa.gov/
} 


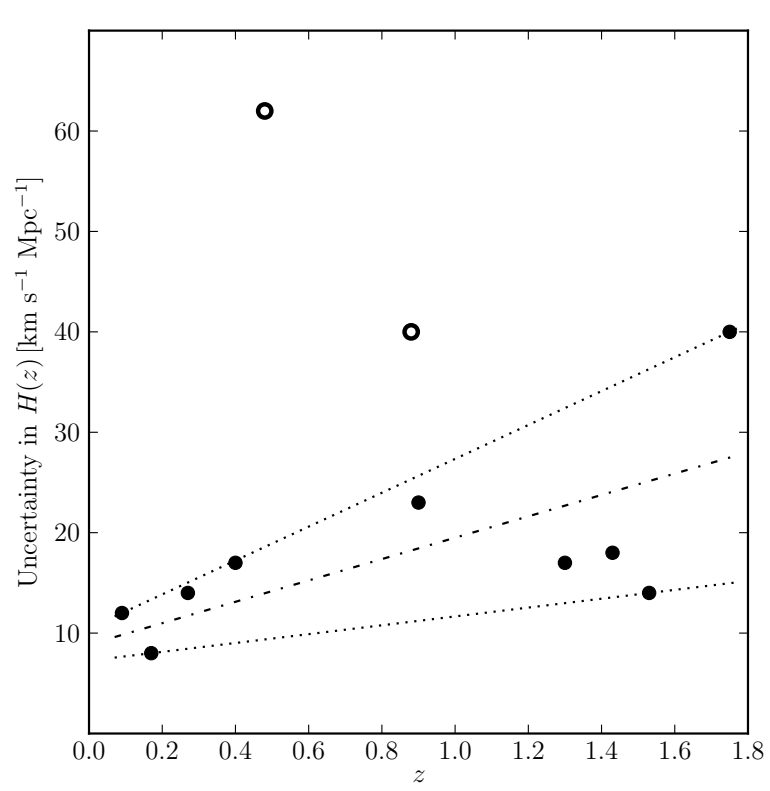

Figure 2. Uncertainties of $H(z)$ in the SJVKS10 dataset. Solid dots and circles represents non-outliers and outliers respectively. Our heuristic bounds $\sigma_{+}$and $\sigma_{-}$are plotted as the two dotted lines. The dash-dotted line shows our estimated mean uncertainty $\sigma_{0}$.

around this difficulty by approaching the problem from a phenomenological point of view.

By inspecting the uncertainties on $H(z)$ in the SJVKS10 dataset (Figure 2), we can see that the general trend of the errors' increasing with $z$ despite the two outliers at $z=0.48$ and 0.88 . Excluding the outliers from the dataset, we find that the uncertainties $\sigma(z)$ are bounded by two straight lines $\sigma_{+}=16.87 z+10.48$ and $\sigma_{-}=4.41 z+7.25$ from above and below respectively. If we believe that future observations would also yield data with uncertainties within the strip bounded by $\sigma_{+}$and $\sigma_{-}$, we can take the midline of the strip $\sigma_{0}=10.64 z+8.86$ as an estimate of the mean uncertainty of future observations. In our code, this is done by drawing a random number $\tilde{\sigma}(z)$ from the Gaussian distribution $N\left(\sigma_{0}(z), \varepsilon(z)\right)$ where $\varepsilon(z)=\left(\sigma_{+}-\sigma_{-}\right) / 4$. The parameter $\varepsilon$ is chosen so that the probability of $\tilde{\sigma}(z)$ falling within the strip is $95.4 \%$.

Having found a method of generating the random uncertainty $\tilde{\sigma}(z)$ for a simulated datapoint, we are able to simulate the deviation from $H_{\text {fid }}$. Namely, we assume that the deviation of the simulated observational value from the fiducial, $\Delta H=H_{\text {sim }}(z)-H_{\text {fid }}(z)$, satisfies the Gaussian distribution $N(0, \tilde{\sigma}(z))$ from which $\Delta H$ can be drawn as a random variable.

Thus a complete procedure of generating a simulated $H(z)$ value at any given $z$ is formed. First, the fiducial value $H_{\text {fid }}(z)$ is calculated from equation (1). After that, a random uncertainty $\tilde{\sigma}(z)$ is drawn using the aforementioned method. This uncertainty is in turn used to draw a random deviation $\Delta H$ from the Gaussian distribution $N(0, \tilde{\sigma}(z))$. The final result of this process is a datapoint $H_{\text {sim }}(z)=H_{\text {fid }}(z)+\Delta H$ with uncertainty $\tilde{\sigma}(z)$.

In addition to the procedures described above, the Hubble constant (in the units of $\mathrm{km} \mathrm{s}^{-1} \mathrm{Mpc}^{-1}$ ) is also taken as a random variable and is drawn from the

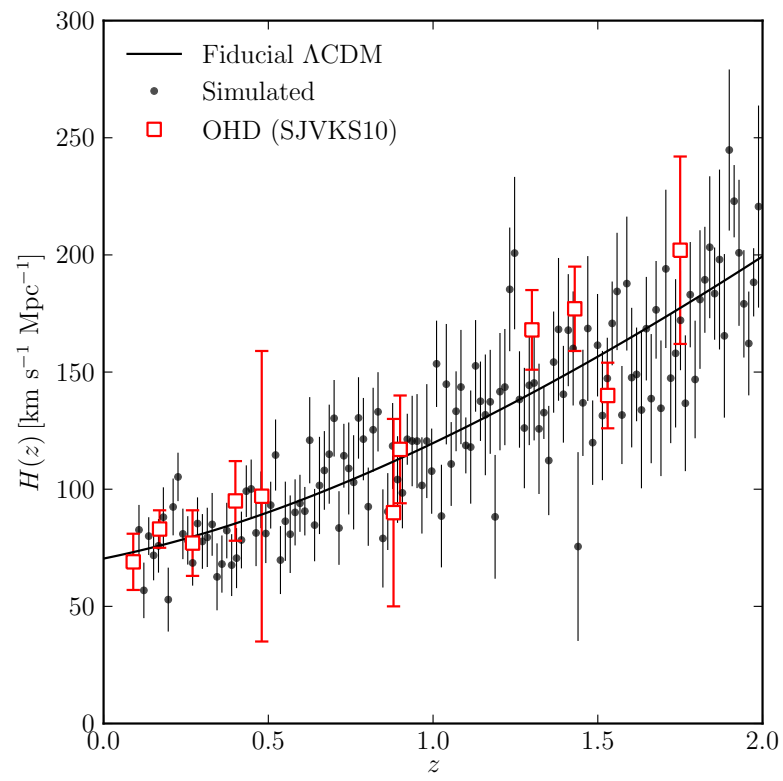

Figure 3. Snapshot of a simulated dataset realized using our method. The uncertainties in the simulated data are modelled phenomenologically after SJVKS10, which is also shown for comparison.

Gaussian distribution $N(70.4,1.4)$ suggested by 7 -year WMAP results when we evaluate the right-hand side of equation (1). We could have fixed $H_{0}$ at a constant value, but as we shall see in Section 3.2 in our analysis we treat $H_{0}$ and $\Omega_{\mathrm{m}}$ quite differently. Namely, $\Omega_{\mathrm{m}}$ is a parameter with a posterior distribution to be inferred, but $H_{0}$ is a nuisance parameter that is marginalized over using some independent measurement results as prior knowledge. This treatment can be found in many works, such as (Stern et al. 2010a) and (Wei 2010). It can be justified by the need to reduce the dimension of the parameter space given limited data, and we usually prioritize other parameters such as $\Omega_{\mathrm{m}}$ over $H_{0}$. Therefore, when generating simulated datasets we sample $H_{0}$ from a random distribution to reflect the uncertainty to be marginalized over. Otherwise, we could have "cheated" by forcing a $\delta$ function prior on $H_{0}$ centered at its fiducial value in the analysis of simulated data and obtain overly optimistic predictions from the simulated data.

The quality of simulated data thus generated is similar to that of SJVKS10. A snapshot realization of this simulation scheme is displayed in Figure 3, where a total of 128 datapoints with $z$ evenly spaced within the range $0.1 \leq z \leq 2.0$.

\subsection{The Evaluation Model}

We use a standard non-flat $\Lambda$ CDM model with a curvature term $\Omega_{\mathrm{k}}=1-\Omega_{\mathrm{m}}-\Omega_{\Lambda}$ to evaluate the qualities of the simulated datasets. In this model, the Hubble parameter is given by

$$
\begin{aligned}
H(z) & =H_{0} \sqrt{\Omega_{\mathrm{m}}(1+z)^{3}+\Omega_{\mathrm{k}}(1+z)^{2}+\Omega_{\Lambda}} \\
& =H_{0} E\left(z ; \Omega_{\mathrm{m}}, \Omega_{\Lambda}\right) .
\end{aligned}
$$

Our model choice is mainly motivated by our desire to reduce unnecessary distractions arising from the intrinsic complexity of certain cosmological models involving dark energy or modified gravitation. 
We perform a standard maximal likelihood analysis using this evaluation model. In our analysis we intend to marginalize the likelihood function over the Hubble parameter $H_{0}$, thus obtaining parameter constraints in the $\left(\Omega_{\mathrm{m}}, \Omega_{\Lambda}\right)$ subspace. This marginalization process also allows us to incorporate a priori knowledge about $H_{0}$ into our analysis.

There is a fair amount of available information from which reasonable priors can be constructed. Samushia \& Ratra (2006) used two different Gaussian priors on $H_{0}$ : one with $H_{0}=73 \pm 3 \mathrm{~km} \mathrm{~s}^{-1} \mathrm{Mpc}^{-1}$ from 3year WMAP data (Spergel et al. 2007), the other with $H_{0}=68 \pm 4 \mathrm{~km} \mathrm{~s}^{-1} \mathrm{Mpc}^{-1}$ by Gott III et al. (2001) (for a discussion of the non-Gaussianity of the error distribution in $H_{0}$ measurements, see Chen et al. 2003). Lin et al. (2009) used $H_{0}=72 \pm 8 \mathrm{~km} \mathrm{~s}^{-1} \mathrm{Mpc}^{-1}$ as suggested by (Freedman et al. 2001). In our work we use a more recent determination of $H_{0}=74.2 \pm 3.6 \mathrm{~km} \mathrm{~s}^{-1} \mathrm{Mpc}^{-1}$ (Riess et al. 2009) as an update to the ones cited above. We also consider a "top-hat" prior, i.e. a uniform distribution in the interval $[50,100]$. Compared with any of the peaked priors above, this prior shows less preference of a particular central value while still characterizing our belief that any value of $H_{0}$ outside the said range is unlikely to be true. Notice that the intrinsic spread of $H_{0}$ involved in the generation of simulated datasets is smaller than either prior chosen in this step, in consistent with the belief that the prior adopted in the estimation of parameters should not be spuriously optimistic.

Having chosen the priors on $H_{0}$, it is straightforward to derive, up to a non-essential multiplicative constant, the posterior probability density function (PDF) of parameters given the dataset $\left\{H_{i}\right\}$ by means of Bayes' theorem:

$$
\begin{array}{r}
P\left(\Omega_{\mathrm{m}}, \Omega_{\Lambda} \mid\left\{H_{i}\right\}\right)=\int P\left(\Omega_{\mathrm{m}}, \Omega_{\Lambda}, H_{0} \mid\left\{H_{i}\right\}\right) \mathrm{d} H_{0} \\
=\int \mathcal{L}\left(\left\{H_{i}\right\} \mid \Omega_{\mathrm{m}}, \Omega_{\Lambda}, H_{0}\right) P\left(H_{0}\right) \mathrm{d} H_{0}
\end{array}
$$

where $\mathcal{L}$ is the likelihood and $P\left(H_{0}\right)$ is the prior PDF of $H_{0}$. Assuming that each measurement in $\left\{H_{i}\right\}$ has Gaussian error distribution of $H(z)$ and is independent from other measurements, the likelihood is then given by

$$
\mathcal{L}\left(\left\{H_{i}\right\} \mid \Omega_{\mathrm{m}}, \Omega_{\Lambda}, H_{0}\right)=\left(\prod_{i} \frac{1}{\sqrt{2 \pi \sigma_{i}^{2}}}\right) \exp \left(-\frac{\chi^{2}}{2}\right),
$$

where the $\chi^{2}$ statistic is defined by

$$
\chi^{2}=\sum_{i} \frac{\left[H_{0} E\left(z_{i} ; \Omega_{\mathrm{m}}, \Omega_{\Lambda}\right)-H_{i}\right]^{2}}{\sigma_{i}^{2}},
$$

and $\sigma_{i}$ 's are the uncertainties quoted from the dataset. The posterior PDF can thus be found by inserting equation (4) and the exact form of $P\left(H_{0}\right)$ into equation (3).

We now show that the integral over $H_{0}$ in equation (3) can be worked out analytically for our $P\left(H_{0}\right)$ choices.

$$
\begin{aligned}
& \text { Gaussian prior. - Let } \\
& \qquad P\left(H_{0}\right)=\frac{1}{\sqrt{2 \pi \sigma_{H}^{2}}} \exp \left[-\frac{\left(H_{0}-\mu_{H}\right)^{2}}{2 \sigma_{H}^{2}}\right] .
\end{aligned}
$$

In this case, equation $\sqrt{3}$ reduces to

$$
\begin{aligned}
P\left(\Omega_{\mathrm{m}},\right. & \left.\Omega_{\Lambda} \mid\left\{H_{i}\right\}\right) \\
& =\frac{1}{\sqrt{A}}\left[\operatorname{erf}\left(\frac{B}{\sqrt{A}}\right)+1\right] \exp \left(\frac{B^{2}}{A}\right)
\end{aligned}
$$

where

$$
\begin{aligned}
& A=\frac{1}{2 \sigma_{H}^{2}}+\sum_{i} \frac{E^{2}\left(z_{i} ; \Omega_{\mathrm{m}}, \Omega_{\Lambda}\right)}{2 \sigma_{i}^{2}}, \\
& B=\frac{\mu_{H}}{2 \sigma_{H}^{2}}+\sum_{i} \frac{E\left(z_{i} ; \Omega_{\mathrm{m}}, \Omega_{\Lambda}\right) H_{i}}{2 \sigma_{i}^{2}},
\end{aligned}
$$

and erf stands for the error function. The form shown in equation (7) is not normalized; all multiplicative constants have been discarded.

Top-hat prior. - The PDF of a uniform distribution over the interval $[x, y]$ can be written as $P\left(H_{0}\right)=\Theta\left(H_{0}-\right.$ $x) \Theta\left(y-H_{0}\right) /(y-x)$ where $\Theta$ denotes the Heaviside unit step function. With this prior on $H_{0}$, equation (3) becomes

$$
\begin{aligned}
P\left(\Omega_{\mathrm{m}},\right. & \left.\Omega_{\Lambda} \mid\left\{H_{i}\right\}\right) \\
& =\frac{U(x, C, D)-U(y, C, D)}{\sqrt{C}} \exp \left(\frac{D^{2}}{C}\right)
\end{aligned}
$$

where

$$
C=\sum_{i} \frac{E^{2}\left(z_{i} ; \Omega_{\mathrm{m}}, \Omega_{\Lambda}\right)}{2 \sigma_{i}^{2}}, \quad D=\sum_{i} \frac{E\left(z_{i} ; \Omega_{\mathrm{m}}, \Omega_{\Lambda}\right) H_{i}}{2 \sigma_{i}^{2}}
$$

and

$$
U(x, \alpha, \beta)=\operatorname{erf}\left(\frac{\beta-x \alpha}{\sqrt{\alpha}}\right) .
$$

The normalization constant has been dropped from formula (8) as well.

\subsection{Figure of Merit}

The posterior probability density functions obtained above put statistical constraints over the parameters. As the dataset $\left\{H_{i}\right\}$ improves in size and quality, the constraints are tightened. To evaluate a dataset's ability of tightening the constrains, a quantified figure of merit (FoM) must be established.

We note that the FoM can be defined arbitrarily as long as it reasonably rewards a tight fit while punishing a loose one. Its definition can be motivated purely statistically, for example the reciprocal hypervolume of the 95\% confidence region in the parameter space (Albrecht et al. 2006). However, a definition that is sensitive to some physically significant structuring of the parameter space can be preferable if our scientific goal requires it (Linder 2006).

In this paper we use a statistical FoM definition similar to the ones of Albrecht et al. (2006), Liu et al. (2008), and Bueno Sanchez et al. (2009). Our FoM is defined to be the area enclosed by the contour of $P\left(\Omega_{\mathrm{m}}, \Omega_{\Lambda} \mid\left\{H_{i}\right\}\right)=\exp \left(-\Delta \chi^{2} / 2\right) P_{\max }$ where $P_{\max }$ is the maximum value of the posterior PDF, and the constant $\Delta \chi^{2}$ is taken to be 6.17 . This value is so chosen that the region enclosed by this contour coincides with the $2 \sigma$ or $95.4 \%$ confidence region if the posterior is Gaussian. 
In the rest of this paper we will use the term " $2 \sigma$ region" to refer to the region defined in this way. The $1 \sigma$ and $3 \sigma$ regions can be defined in the same manner by setting the constant $\Delta \chi^{2}$ to 2.3 and 11.8 respectively. It is important to bear in mind that our definition of " $n \sigma$ regions" are motivated by nomenclature brevity rather than mathematical concreteness, since the posterior PDFs (eqs. 7] and 8) are manifestly non-Gaussian.

We make two further remarks on our FoM definition. First, since our definition of FoM is statistical rather than physical, we do not exclude the unrealistic parts of the confidence regions from the area. Second, the FoM by our definition is obviously invariant under the multiplication of $P\left(\Omega_{\mathrm{m}}, \Omega_{\Lambda} \mid\left\{H_{i}\right\}\right)$ by a positive constant, which justifies the omission of normalization constants in Section 3.2 .

\section{RESULTS FROM THE SIMULATED DATASETS}

Using the method described in Section 3.1. we generate 500 realizations of the simulated $H(z)$ dataset. Each realization contains 128 datapoints evenly spaced in the redshift range $0.1 \leq z \leq 2.0$. From each realization, successively shrinking subsamples of 64,32 , and 16 datapoints are randomly drawn. These subsamples are then used in conjunction with the full OHD set to obtain their respective figures of merit.

The figures of merit are naturally divided into 4 groups by the size of the simulated subsample used in the calculation. For each group, median and median absolute deviation (MAD) statistics are calculated. The median and the MAD are used to represent the central value and spread of FoM data respectively, and they are used in preference to the customary pair of the mean and the standard deviation, because they are less affected by egregious outliers (see NIST 2003, Chapter 1.3.5.6). The outliers mainly arise from "worst case" realizations of simulated $H(z)$ data, for example one with a large amount of datapoints deviating too much (or too little) from the fiducial model.

To compare the parameter constraining abilities of our simulated $H(z)$ datasets with those of SNIa data, we have also fitted our evaluation model (eq. 22) to the ConstitutionT dataset which is a subset of the Constitution redshift-distance dataset (Hicken et al. 2009b) deprived of outliers that account for internal tensions (Wei 2010). It is worthwhile to point out that the prior of $H_{0}$ used in the SNIa fitting procedure is fundamentally different from either one discussed in Section 3.2. Namely, when SNIa data is used, the parameter $H_{0}$ and the intrinsic absolute magnitude of SNIa, $M_{0}$, are combined into one "nuisance parameter" $M=M_{0}-5 \lg H_{0}$, and is marginalized over under the assumption of a flat prior over $(-\infty,+\infty)$ (Perlmutter et al. 1999). This discrepancy should be kept in mind when comparing or combining SNIa and $H(z)$ datasets, and we hope it could be closed in the future by better constraints, either theoretical or observational, on $M_{0}$.

Our main results are show in Figure 4. As one may intuitively assume, the median FoM increases with the size of the dataset. We find that the data subset with 64 simulated datapoints leads to a FoM of $8.6 \pm 0.7$ under the Gaussian prior on $H_{0}$. This median FoM already surpasses that of ConstitutionT. However, the top-hat prior leads to significantly lower FoM. Under the top-hat prior we used, as many as 128 simulated datapoints are

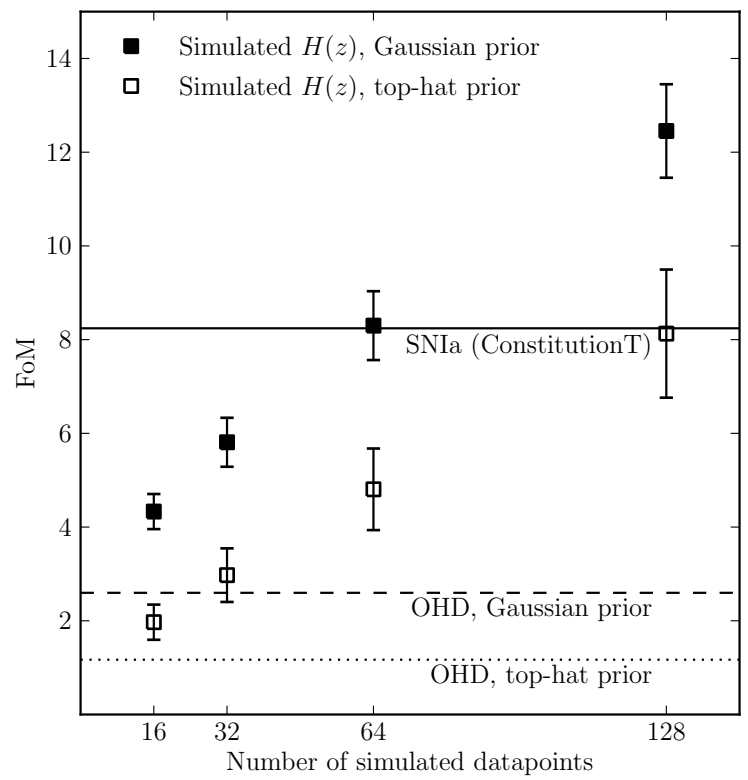

Figure 4. Figures of merit from each group found by combining all 500 realizations. FoM medians are plotted against the sizes of the simulated subsample, and median absolute deviations are shown as error bars. Horizontal lines across the figure marks the figures of merit of purely observational datasets.

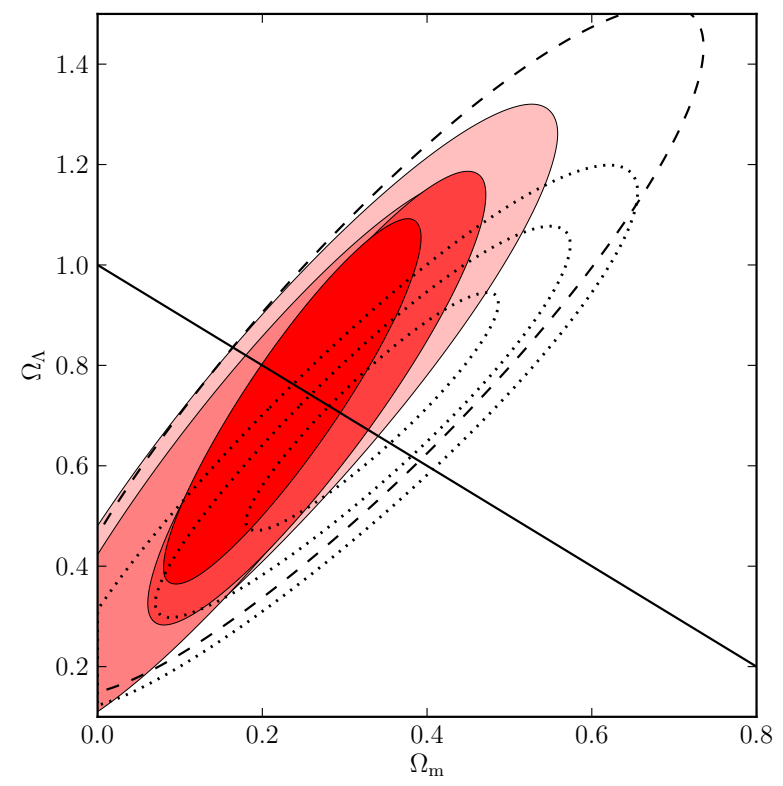

Figure 5. Confidence regions in the $\left(\Omega_{\mathrm{m}}, \Omega_{\Lambda}\right)$ parameter subspace calculated using the snapshot realization shown in Figure 3 The Gaussian prior $H_{0}=74.2 \pm 3.6 \mathrm{~km} \mathrm{~s}^{-1} \mathrm{Mpc}^{-1}$ is assumed. The shaded regions, from lighter to darker, correspond to the $2 \sigma$ constraints obtained from the data subsets containing 16, 32, 64, and 128 simulated $H(z)$ datapoints respectively. For comparison, the $2 \sigma$ region from pure OHD alone, with the same Gaussian prior on $H_{0}$, is plotted in the dashed contour. The three dotted contours are the 1,2 , and $3 \sigma$ constraints from the ConstitutionT SNIa dataset. The solid straight line signifies the boundary of $\Omega_{\mathrm{k}}=0$.

needed to reaches the FoM level of ConstitutionT.

The degeneracy of confidence regions obtained from $H(z)$ data in the $\left(\Omega_{\mathrm{m}}, \Omega_{\Lambda}\right)$ parameter subspace is shown in Figure 5. Because of this degeneracy, $H(z)$ datasets cannot be used alone to constrain $\Omega_{\mathrm{k}}$ well. This degen- 
erate behavior is similar to that of SNIa data.

\section{FISHER MATRIX ANALYSIS OF FUTURE DATA}

The simulated data used in Section 3 are based on the quality of currently available measurements, and we have tried not to be too optimistic about their uncertainties. However, we have reasons to expect an increase in the quality of future $H(z)$ data. First, Crawford et al. (2010) analysed the observational requirement of measuring $H(z)$ to $3 \%$ at intermediate redshifts with agedating. Second, the Baryon Oscillation Spectroscopic Survey (BOSS ${ }^{4}$ ) is designed to constrain $H(z)$ with $2 \%$ precision at redshifts $z \approx 0.3$ and 0.6 by measuring $\mathrm{BAO}$ imprints in the galaxy field, and at $z \approx 2.5$ using the Lyman- $\alpha$ absorption spectra of quasars.

By incorporating these specifications of future data, we can estimate their expected figures of merit using the Fisher matrix forecast technique (Dodelson et al. 1997). The $3 \times 3$ Fisher matrix $\boldsymbol{F}$ is calculated from equation (5) with the $\sigma_{i}$ 's determined by future data specifications, and the evaluation of matrix elements is made at the fiducial parameter values (see Section 3.1). The matrix elements are the second partial derivatives of $\chi^{2}$ with respect of the parameters:

$$
F_{i j}=\frac{1}{2} \frac{\partial^{2} \chi^{2}}{\partial \theta_{i} \partial \theta_{j}},
$$

where the $\theta_{i}$ 's are the parameters, namely $\left(\Omega_{\mathrm{m}}, \Omega_{\Lambda}, H_{0}\right)$. Notice that we use the word "Fisher matrix" only loosely. The second derivative matrix defined by equation 90 is not the Fisher matrix in the strict sense, but the ideas are intimately related (see Dodelson 2003).

In order to obtain the FoM in the 2-dimensional parameter space of $\left(\Omega_{\mathrm{m}}, \Omega_{\Lambda}\right)$, we must marginalize over $H_{0}$. We adopt the Gaussian prior on $H_{0}$ with $\sigma_{H}=$ $3.6 \mathrm{~km} \mathrm{~s}^{-1} \mathrm{Mpc}^{-1}$, which is the same as the one used in Section 3.2. Gaussian marginalization is performed using a straightforward modification of the projection technique from (Dodelson 2003) and (Press et al. 2007). A more general and detailed analysis of this problem was made by Taylor \& Kitching (2010), but for our purpose, simply adding $1 / \sigma_{H}^{2}$ to $F_{33}$ and then projecting onto the first two dimensions will do the work. This is applicable when the mean of the prior $H_{0}$ is close to the fiducial value, and we have numerically verified the validity of this approximation in our work.

Denoting the marginalized Fisher matrix by $\widetilde{\boldsymbol{F}}$, the iso$\Delta \chi^{2}$ contour in the parameter space is approximated by the quadratic equation

$$
(\Delta \boldsymbol{\theta})^{T} \widetilde{\boldsymbol{F}} \Delta \boldsymbol{\theta}=\Delta \chi^{2}
$$

where $\Delta \boldsymbol{\theta}=\boldsymbol{\theta}-\boldsymbol{\theta}_{\mathrm{fid}}$ is the parameters' deviation from the fiducial value, and $\Delta \chi^{2}$ is our chosen constant 6.17 in Section 3.3. By construction, $\widetilde{\boldsymbol{F}}$ is positive-definite 5 therefore the above equation describes an ellipse. Its enclosed area is simply $A=\pi / \sqrt{\operatorname{det}\left(\widetilde{\boldsymbol{F}} / \Delta \chi^{2}\right)}$, therefore

\footnotetext{
4 http://www.sdss3.org/boss.php

5 This can be proved using the positive-definiteness conditions of the Schur complement (Puntanen \& Styan 2005).
}

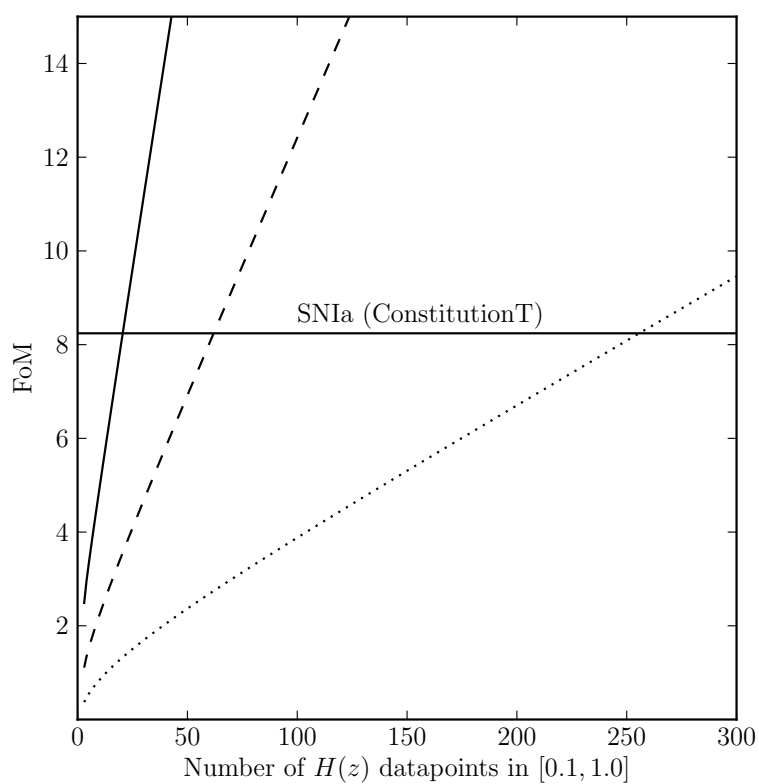

Figure 6. Predicted FoM using the error model of (Crawford et al. 2010). The dependence of FoM on the number of measurements is shown for three cases: the solid, dashed, and dotted lines are for $3 \%, 5 \%$, and $10 \%$ relative error respectively. The horizontal line across the figure shows the FoM of ConsitutionT for comparison.

we can estimate the FoM by

$$
\mathrm{FoM}=\frac{1}{A}=\frac{1}{\pi} \sqrt{\operatorname{det}\left(\frac{1}{\Delta \chi^{2}} \widetilde{\boldsymbol{F}}\right)} .
$$

In our setup, we assume that the relative error of $H(z)$ anticipated by Crawford et al. (2010) could be globally achieved within the redshift interval $0.1 \leq z \leq 1.0$. Under this assumption, we can estimate how many datapoints will be needed to reach the ConstitutionT FoM using the Fisher matrix method discussed above. We chose not to incorporate the available data so that we can work with the simple error model specified by future data.

Our main results are shown in Figures 6 and 7 . For relative $H(z)$ errors of $3 \%, 5 \%$, and $10 \%$, the required number of measurements are 21,62, and 256 respectively. This number $N$ increases steeply as the relative error increases, as shown in Figure 7.

We can also apply the Fisher matrix analysis to future BOSS data. We find BOSS alone gives $\mathrm{FoM} \approx 15$. This promising result is a combined consequence of its high precision and extended redshift coverage. In Figure 8 we plot the Fisher matrix forecast of the confidence regions.

\section{CONCLUSION AND DISCUSSIONS}

We have explored the possibility of using OHD as an alternative to SNIa redshift-distance data in the sense of offering comparable or higher FoM. By using simulated $H(z)$ datasets with an empirical error model similar to that of current age-dating data, we show that more than 60 future measurements of $H(z)$ in the redshift range $0 \leq z \leq 2$ could be needed to acquire parameter constraints comparable with those obtained from SNIa datasets like ConstitutionT. In addition, precise and ac- 


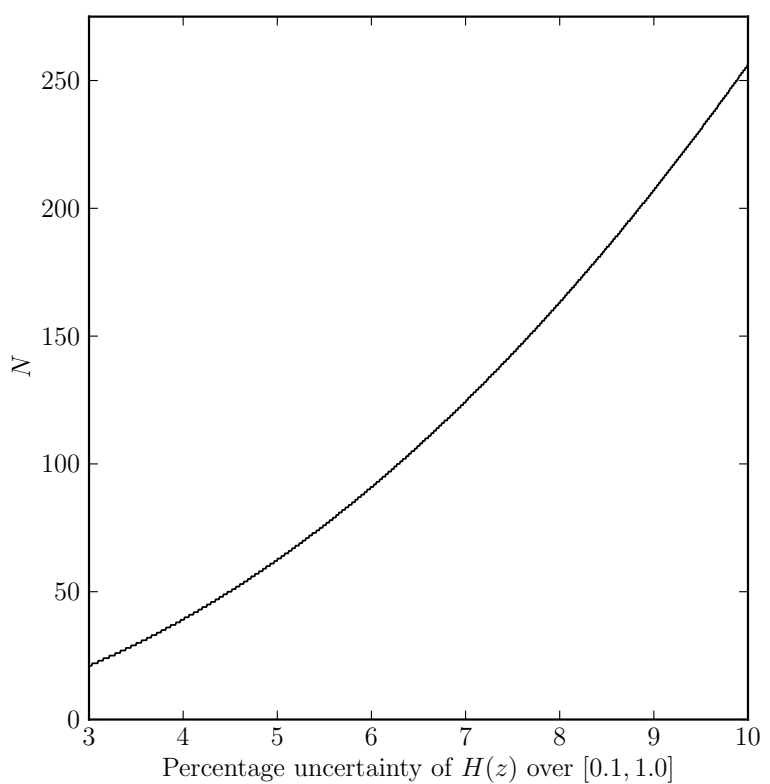

Figure 7. Number of measurements required to match ConstitutionT, $N$, as a function of the relative error of $H(z)$. The curve turns upward steeply when the error is large.

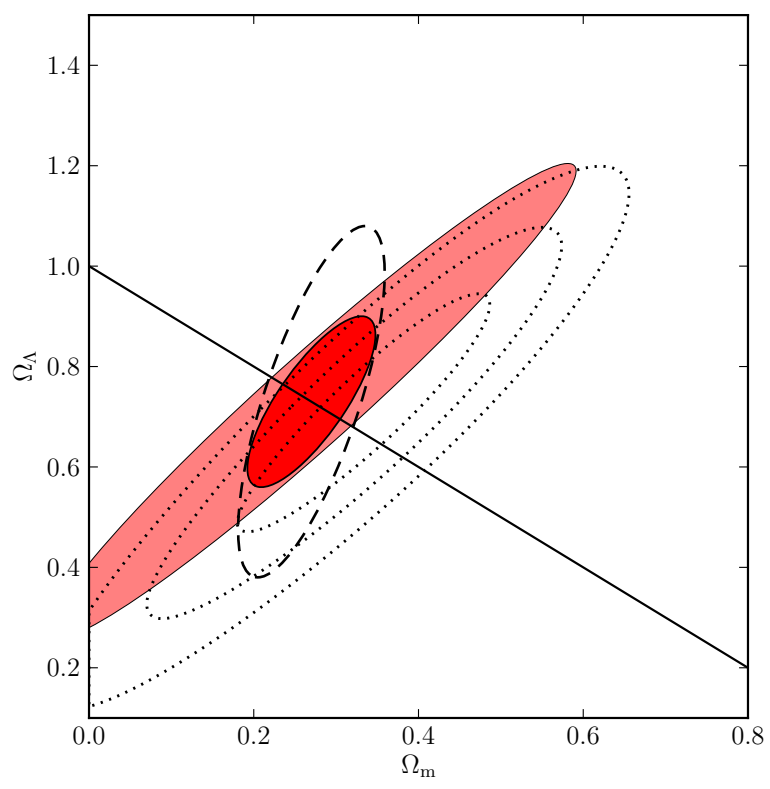

Figure 8. Same as Figure 4 but for the forecast of future data. The light shaded region is the $2 \sigma$ region from the 21 age-dating measurements with relative error $3 \%$. The unfilled region bounded by the dashed contour is the $2 \sigma$ region from BOSS $H(z)$. The joint $2 \sigma$ region of BOSS and age-dating is shown as the inner and darker shaded area. Like in Figure 4 1, 2, and $3 \sigma$ constraints from ConstitutionT are shown as dotted contours for comparison.

curate determination of $H_{0}$ is crucial for improving the FoM obtained from OHD, and a broad prior on $H_{0}$ leads to lower FoM. When we progressively lower the error of future measurements to $3 \%$ as discussed by (Crawford et al. 2010), we estimate that $\sim 60$ measurements in the shorter redshift interval $[0.1,1.0]$ will be needed to achieve the same result. In summary, we give an affirmative answer to the first of the two questions raised in Section 1 and a semi-quantitative answer to the second.
Our result furthers a conclusion of Lin et al. (2009) and Carvalho et al. (2008), namely, that OHD play aImost the same role as that of SNIa for the joint constraints on the $\Lambda \mathrm{CDM}$ model. We have shown that the OHD set alone is potentially capable to be used in place of current SNIa datasets if it is large enough. We note that our forecast of $\mathrm{OHD}$ data requirement is competitive in the sense of observational cost compared with supernova observations. Throughout our analysis, we used the ConstitutionT dataset used as a standard of FoM. This dataset is a subset of the Constitution compilation, a combination of the ground-based CfA3 supernova observations (Hicken et al. 2009a) and Union, a larger compilation of legacy supernovae and space-based observations (Kowalski et al. 2008). The CfA3 sample alone requires 10 nights for each of the 185 supernovae observed. On the other hand, current OHD from age-dating does not require space-based observations. As described in detail by Stern et al. (2010b), 24 galaxy cluster containing target chronometer galaxies were obtained in only two nights using the Keck I telescope. In (Crawford et al. 2010), it was estimated that the South African Large Telescope (SALT) is capable to measure $H(z)$ to $3 \%$ at an individual redshift in $\sim 180$ hours.

Admittedly, our approach to the problem is tentative as well as model-dependent. The conclusion is reached under the assumption of the fiducial $\Lambda$ CDM model (eq. [1]), the model of uncertainties described in Sections 3.1 and 5, as well as the evaluation model of Section 3.2. Therefore, care must be taken not to extrapolate our conclusions well beyond those assumptions, especially when used in the planning of observations.

Even if our uncertainty models fit real-world observations well, in practice the results may be less promising than what we suggested in this paper. We assumed a fairly deep redshift coverage which may be difficult to reach by current observations (see Stern et al. 2010b, for the redshift distribution of LRGs used to deduce SJVKS10). Ultimately, the FoM from OHD may be limited by our ability to select enough samples for future observation and the redshift distribution of these samples, rather than by the relative error of each individual measurement. Fortunately, the BOSS project could extend $H(z)$ measurement into deeper redshift. Also, It is worth noting that the proposed Sandage-Loeb observational plan (Corasaniti et al. 2007; Zhang et al. 2010. Araújo \& Stoeger 2010) could be used to extend our knowledge of cosmic expansion into the even deeper redshift realm of $2 \leq z \leq 5$ by measuring the secular variation of cosmological redshifts of the Lyman- $\alpha$ forest with future high-resolution, space-based spectroscopic instruments (see (Sandage 1962) and (Loeb 1998) for the foundations of the test).

Finally, we note that future CMB observation programs, such as the Atacama Cosmology Telescop 6 may be able to identify more than 2000 passively evolving galaxies up to $z \approx 1.5$ via the Sunyaev-Zel'dovich effect, and their spectra can be analyzed to yield age measurements that will yield approximately $1000 H(z)$ determinations with $15 \%$ error (Simon et al. 2005). This promises a future data capacity an order of magnitude more than what we have estimated to be enough to match

6 http://www.physics.princeton.edu/act/index.html 
current SNIa datasets. Combining this prospect with future high- $z$, high-accuracy $H(z)$ determinations from $\mathrm{BAO}$ observations, it is reasonable to expect that the OHD will play an increasingly important role in the future study of the expansion history of the universe and cosmological parameters.

We thank the anonymous referee whose suggestions greatly helped us improve this paper. CM is grateful to Chen-Tao Yang for useful discussions. This work was supported by the National Science Foundation of China (Grants No. 10473002), the Ministry of Science and Technology National Basic Science program (project 973) under grant No. 2009CB24901, the Fundamental Research Funds for the Central Universities.

\section{REFERENCES}

Albrecht, A. et al. 2006, preprint, arXiv:astro-ph/0609591

Aldering, G. et al. 2004, preprint, arXiv:astro-ph/0405232

Araújo, M. E., \& Stoeger, W. R. 2010, Phys. Rev. D, 82, 123513, arXiv:1009.2783

Blake, C., \& Glazebrook, K. 2003, ApJ, 594, 665, arXiv:astro-ph/0301632

Bueno Sanchez, J. C., Nesseris, S., \& Perivolaropoulos, L. 2009 J. Cosmol. Astropart. Phys., 11, 29, arXiv:0908.2636

Carvalho, F. C., Santos, E. M., Alcaniz, J. S., \& Santos, J. 2008, J. Cosmol. Astropart. Phys., 9, 8, arXiv:0804.2878

Chen, G., Gott III, J. R., \& Ratra, B. 2003, PASP, 115, 1269 arXiv:astro-ph/0308099

Corasaniti, P., Huterer, D., \& Melchiorri, A. 2007, Phys. Rev. D, 75, 062001, arXiv:astro-ph/0701433

Crawford, S. M., Ratsimbazafy, A. L., Cress, C. M., Olivier, E. A., Blyth, S., \& van der Heyden, K. J. 2010, MNRAS, 406, 2569, arXiv: 1004.2378

Dodelson, S. 2003, Modern Cosmology (San Diego, CA: Academic Press)

Dodelson, S., Kinney, W. H., \& Kolb, E. W. 1997, Phys. Rev. D, 56, 3207, arXiv:astro-ph/9702166

Eisenstein, D. J. et al. 2005, ApJ, 633, 560, arXiv:astro-ph/0501171

Freedman, W. L. et al. 2001, ApJ, 553, 47, arXiv:astro-ph/0012376

Gaztañaga, E., Cabré, A., \& Hui, L. 2009, MNRAS, 399, 1663, arXiv:0807.3551

Ghirlanda, G., Ghisellini, G., Lazzati, D., \& Firmani, C. 2004, ApJ, 613, L13, arXiv:astro-ph/0408350

Gott III, J. R., Vogeley, M. S., Podariu, S., \& Ratra, B. 2001, ApJ, 549, 1, arXiv:astro-ph/0006103

Hicken, M. et al. 2009a, ApJ, 700, 331, arXiv:0901.4787

Hicken, M., Wood-Vasey, W. M., Blondin, S., Challis, P., Jha, S., Kelly, P. L., Rest, A., \& Kirshner, R. P. 2009b, ApJ, 700, 1097, arXiv:0901.4804

Huterer, D. 2009, Nuclear Physics B Proceedings Supplements, 194, 239

Jimenez, R., \& Loeb, A. 2002, ApJ, 573, 37, arXiv:astro-ph/0106145

Jimenez, R., Verde, L., Treu, T., \& Stern, D. 2003, ApJ, 593, 622, arXiv:astro-ph/0302560
Kim, A. G., Linder, E. V., Miquel, R., \& Mostek, N. 2004, MNRAS, 347, 909, arXiv:astro-ph/0304509

Komatsu, E. et al. 2010, ApJS, submitted, arXiv:1001.4538

Kowalski, M. et al. 2008, ApJ, 686, 749, arXiv:0804.4142

Li, H., Xia, J.-Q., Liu, J., Zhao, G.-B., Fan, Z.-H., \& Zhang, X. 2008, ApJ, 680, 92, arXiv:0711.1792

Li, M., Li, X.-D., Wang, S., \& Zhang, X. 2009

J. Cosmol. Astropart. Phys., 6, 36, arXiv:0904.0928

Liang, N., Wu, P., \& Zhang, S. N. 2010, Phys. Rev. D, 81, 083518, arXiv:0911.5644

Lin, H., Hao, C., Wang, X., Yuan, Q., Yi, Z.-L., Zhang, T.-J., \& Wang, B.-Q. 2009, Mod. Phys. Lett. A, 24, 1699, arXiv:0804.3135

Linder, E. V. 2006, Astropart. Phys., 26, 102 , arXiv:astro-ph/0604280

Liu, D.-J., Li, X.-Z., Hao, J., \& Jin, X.-H. 2008, MNRAS, 388 275, arXiv:0804.3829

Loeb, A. 1998, ApJ, 499, L111, arXiv:astro-ph/9802112

Mignone, C., \& Bartelmann, M. 2008, A\&A, 481, 295 , arXiv:0711.0370

NIST. 2003, NIST/SEMATECH e-Handbook of Statistical Methods (Gaithersburg, MD: National Institute of Standards and Technology)

http://www.itl.nist.gov/div898/handbook/index.htm

Percival, W. J. et al. 2010, MINKAS, 401, 2148, arXiv:0907.1660

Perlmutter, S. et al. 1999, ApJ, 517, 565, arXiv:astro-ph/9812133

Press, W. H., Teukolsky, S. A., Vetterling, W. T., \& Flannery, B. P. 2007, Numerical Recipes: the Art of Scientific Computing, 3rd edn. (Cambridge: Cambridge Univ. Press)

Puntanen, S., \& Styan, G. P. H. 2005, in Numerical Methods and Algorithms, Vol. 4, The Schur Complement and Its Applications, ed. C. Brezinski \& F. Zhang (New York: Springer), 163-226

Riess, A. G. et al. 1998, AJ, 116, 1009, arXiv:astro-ph/9805201 . 2009, ApJ, 699, 539, arXiv:0905.0695

Samushia, L., \& Ratra, B. 2006, ApJ, 650, L5, arXiv:astro-ph/0607301

Sandage, A. 1962, ApJ, 136, 319

Seo, H.-J., \& Eisenstein, D. J. 2005, ApJ, 633, 575 arXiv:astro-ph/0507338

Shafieloo, A., Alam, U., Sahni, V., \& Starobinsky, A. A. 2006, MNRAS, 366, 1081, arXiv:astro-ph/0505329

Simon, J., Verde, L., \& Jimenez, R. 2005, Phys. Rev. D, 71, 123001, arXiv:astro-ph/0412269

Spergel, D. N. et al. 2007, ApJS, 170, 377 arXiv:astro-ph/0603449

Stern, D., Jimenez, R., Verde, L., Kamionkowski, M., \& Stanford, S. A. 2010a, J. Cosmol. Astropart. Phys., 2, 8, arXiv:0907.3149

Stern, D., Jimenez, R., Verde, L., Stanford, S. A., \& Kamionkowski, M. 2010b, ApJS, 188, 280, arXiv:0907.3152

Taylor, A. N., \& Kitching, T. D. 2010, MNRAS, 408, 865, arXiv:1003.1136

Wang, Y., \& Tegmark, M. 2004, Phys. Rev. Lett., 92, 241302, arXiv:astro-ph/0403292

. 2005, Phys. Rev. D, 71, 103513, arXiv:astro-ph/0501351

Wei, H. 2010, Phys. Lett. B, 687, 286, arXiv:0906.0828

Yang, X.-J., \& Chen, D.-M. 2009, MNRAS, 394, 1449 arXiv:0812.0660

Yi, Z.-L., \& Zhang, T.-J. 2007, Mod. Phys. Lett. A, 22, 41 , arXiv:astro-ph/0605596

Zhang, J., Zhang, L., \& Zhang, X. 2010, Phys. Lett. B, 691, 11, arXiv:1006.1738 